\title{
La aceptación de las TIC por profesorado universitario: Conocimiento, actitud y practicidad
}

\author{
ICT Acceptance by University Professors: Knowledge, Attitude, and Practicality
}

\section{A aceitação das TIC pelos professores universitários: conhecimentos, atitudes e praticidade}

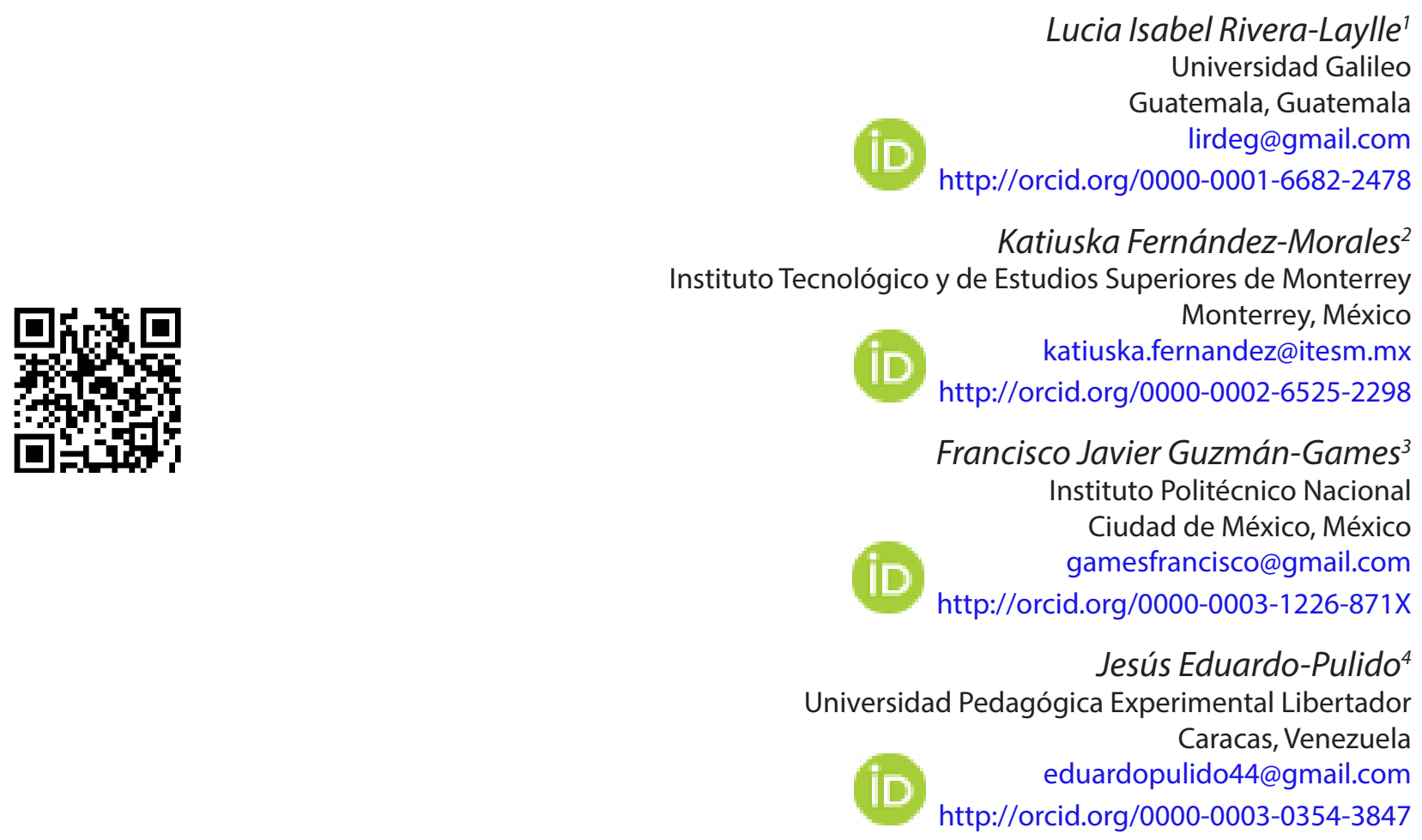

\footnotetext{
${ }^{1}$ Magister en Docencia Universitaria de la Universidad Rafael Landivar de Guatemala y Magister en Dirección y Producción de E-learning de la Universidad Galileo de Guatemala, Guatemala. Labora en la Universidad Galileo y Universidad Mariano Galvez de Guatemala.

${ }^{2}$ Doctora en Investigación Educativa de la Universidad Veracruzana, México. Labora en el Instituto Tecnológico y de Estudios Superiores de Monterrey (ITESM).

${ }^{3}$ Maestro en Investigación Educativa de la Universidad Veracruzana, México. Labora en el Instituto Politécnico Nacional (IPN).

${ }^{4}$ Doctor Honoris Causa y Magíster Scientiarum en Educación de la Universidad Pedagógica Experimental Libertador, Venezuela. Labora en la Universidad Pedagógica Experimental Libertador-Instituto de Mejoramiento Profesional del Magisterio (UPEL-IMPM).
} 
doi: http://dx.doi.org/10.15359/ree.21-3.6

URL: http://www.una.ac.cr/educare

CORREO: educare@una.cr

Recibido 18 de marzo de 2016 • Corregido 9 de junio de 2017 • Aceptado 3 de agosto de 2017 Received 18 de marzo de 2016 • Revised 9 de junio de 2017 • Accepted 3 de agosto de 2017 Recebido 18 de marzo de 2016 • Revisado 9 de junio de 2017 • Aprovado 3 de agosto de 2017

Resumen: El objetivo de esta investigación es analizar la aceptación de las tecnologías de la información y la comunicación (TIC) como herramientas de apoyo para la docencia en universidades privadas de Guatemala. La investigación es cuantitativa, de tipo descriptivo y transversal. Se llevó a cabo con docentes que imparten cursos de grado y posgrado en la modalidad escolarizada y que utilizan entornos virtuales de aprendizaje. Se usó un cuestionario con el fin de medir la "aceptación tecnológica" (dimensión) a partir de tres subdimensiones: conocimiento, actitud y practicidad. Los datos colectados se analizaron mediante la estadística descriptiva. Los resultados se interpretaron con base en el "Modelo de aceptación tecnológica" (Davis, 1989; Venkatesh y Bala, 2008; Venkatesh, Morris, Davis y Davis, 2003). Se encontró que personal docente está convencido de la utilidad de las TIC debido a la variedad de recursos que proporcionan para desarrollar prácticas escolares ajustadas a las necesidades de formación y a las necesidades derivadas de la sociedad del conocimiento, pero "rara vez" supervisan el uso correcto de estas herramientas en el estudiantado y "nunca" buscan la asesoría de personal experto para reforzar las competencias estudiantiles en el manejo de las tecnologías mencionadas.

Palabras claves: Tecnología de la información;tecnología de la comunicación; docente; conocimiento, actitud.

Abstract: The aim of this research is to analyze the acceptance of the Information and Communications Technology as tools to support the practice of teaching in private universities in Guatemala. It is a quantitative, descriptive, and transversal research study. It has been carried out with professors of degree and postgraduate courses in presential mode and with virtual environments of learning. A questionnaire was used to measure the "technology acceptance" (dimension) from three subdimensions: knowledge, attitude, and practicality. To analyze the data collected, we used descriptive statistics. The results were interpreted based on the "Technology Acceptance Model" (Davis, 1989; Venkatesh et al., 2003; Venkatesh y Bala, 2008). It was found that teachers are convinced of the usefulness of ICT, due to the variety of resources provided to develop school practices, tailored to the training needs and the needs arising from the knowledge society. However, professors "rarely" supervise the correct use of these tools by the student and "never" seek expert advice on the subject.

Keywords: Information technology; communication technology; teachers, knowledge; attitudes.

Resumo: O objetivo desta pesquisa é analisar a aceitação das tecnologias da informação e da comunicação (TIC) como ferramentas de apoio para o ensino nas universidades particulares da Guatemala. A pesquisa é quantitativa, descritiva e transversal. Foi realizada com professores que ensinam em cursos de graduação e pós-graduação na modalidade de escola e utilizam ambientes virtuais de aprendizagem. Através de um questionário elaborado para medir a "aceitação tecnológica" (como dimensão), se divide em três subdimensões: conhecimento, atitude e praticidade. Depois de coletar os dados, estes foram analisados utilizando estatística descritiva. Os resultados foram interpretados com base no "modelo de aceitação tecnológica" (Davis, 1989; Venkatesh e Bala, 2008; Venkatesh, Morris, Davis e Davis, 2003). Verificou-se que a equipe docente está convencida da utilidade das TIC por causa da variedade de recursos fornecidos para

2 Lucia Isabel Rivera-Laylle, Katiuska Fernández-Morales, Francisco Javier Guzmán-Games y Jesús Eduardo-Pulido

Los artículos de la Revista Electrónica Educare del Centro de Investigación y Docencia en Educación de la Universidad Nacional, Costa Rica, se comparten bajo términos de la Licencia Creative Commons: Reconocimiento, No Comercial, Sin Obra Derivada 3.0 Costa Rica. Las autorizaciones adicionales a las aquí delimitadas se pueden obtener en el correo: educare@una.cr 


\begin{abstract}
desenvolver práticas escolares adaptadas às necessidades de formação e as necessidades decorrentes da sociedade do conhecimento. Mas "raramente" o uso adequado dessas ferramentas, por parte dos estudantes, são supervisionadas e"nunca" procuram o aconselhamento de funcionários especializados para melhorar as competências dos alunos em lidar com as tecnologias mencionadas.
\end{abstract}

Palavras-chave: Tecnologia da Informação; tecnologia de comunicação; ensino; conhecimento, atitude.

\title{
Introducción
}

En la sociedad del conocimiento del siglo XXI es imperativo que quienes ejercen la docencia estén capacitados en conocimientos, habilidades y actitudes para el uso eficiente e inteligente de las TIC. Este tipo de perfil docente es demandado de forma categórica por el sector productivo que funciona con base en el capitalismo y neoliberalismo. A modo de contexto, el sistema capitalista prevalece en América Latina (y Guatemala). Así que, la viabilidad y pertinencia de las instituciones educativas latinoamericanas depende, en gran medida, de su capacidad para desarrollar programas de formación en consonancia con la estructura social capitalista que dicta la lógica del mercado de trabajo.

América Latina es una región de las menos competitivas a nivel mundial cuando se trata de la capacitación del profesorado en materia de TIC. En particular, Guatemala es uno de los países más rezagados de esta región. El porcentaje de docentes que ha tomado cursos sobre TIC en Guatemala es del $6 \%$, no muy diferente al de otros países en desarrollo como Nicaragua y Paraguay que presentan $17 \%$ y $10 \%$ respectivamente, pero muy dispar comparado con países como Chile (90\%), Perú (82\%), Colombia (61\%) o Costa Rica (60\%). Se estima que apenas poco más de un tercio del total de docentes de Latinoamérica ha recibido capacitación en el uso de TIC (Sistema de Información de Tendencias Educativas en América Latina, SITEAL, 2014).

En Guatemala, el sistema educativo se ha caracterizado por tratar de estar a la vanguardia en materia de tecnologías de la información y la comunicación con base en el establecimiento de una agenda digital (Kats, 2009). El Ministerio de Educación de este país ha implementado cursos de capacitación para docentes de las zonas urbanas y rurales, los que no han tenido resultados significativos (SITEAL, 2014).

Aunque se han hecho grandes esfuerzos, las TIC aún no se han posicionado como una posibilidad auténtica para llevar educación a todos los rincones del país, por ejemplo, el paso de la presencialidad hacia la virtualidad o la combinación de ambas modalidades, en Guatemala no ha sido fácil. Al respecto, afirma Tobar (2010) que solo en el nivel de posgrados hay programas que operan de manera formal en la modalidad de educación en línea. Asimismo, sostiene que en el resto del sistema universitario no hay ofertas para pregrado en esta modalidad. Sin embargo, confirma que el profesorado universitario utiliza las plataformas tecnológicas para complementar sus actividades académicas, lo que ha dado apertura a la educación mixta. 
doi: http://dx.doi.org/10.15359/ree.21-3.6

URL: http://www.una.ac.cr/educare

CORREO: educare@una.cr

En Guatemala la educación mediada por tecnología no está desarrollada en todo su potencial, es decir, hay muchas áreas de oportunidad, porque sigue pendiente desde la estructuración de programas que permitan el acceso a la educación superior a personas en situación de desventaja, hasta la elaboración de propuestas que culminen en políticas públicas para reglamentar el desarrollo de la educación en línea o de la educación mixta.

Guatemala tiene brechas no solo digitales sino también educativas. Cuenta con una población de 14,636,487 habitantes: $48.9 \%$ hombres y $51.1 \%$ mujeres. La población comprendida en el rango de edad entre 15 y 24 años cuenta con un promedio de escolaridad de 6.5 años aprobados (Instituto Nacional de Estadística Guatemala, 2015). Esto significa que la educación superior es para una élite que puede acceder a una institución pública o que puede pagar una institución privada. El sistema educativo de Guatemala se ha visto en la necesidad de innovar el proceso de enseñanza-aprendizaje a partir del uso racional de las TIC. Por ello, las universidades han implementado diversas políticas que buscan garantizar la formación, capacitación y actualización a través de programas institucionales orientados a fortalecer el desempeño docente.

\section{Antecedentes}

El profesorado usa diferentes herramientas basadas en las tecnologías de información y comunicación (TIC), que ofrece el Estado a través de plataformas educativas a fin de fortalecer las metodologías de enseñanza en diversos ambientes de aprendizaje (SITEAL, 2014). No obstante, la investigación educativa ha demostrado que el personal docente tiene diversas limitaciones cuando se trata de utilizar las TIC con fines formativos, por ejemplo; resistencia de uso, falta de capacitación, necesidad de infraestructura tecnológica y mantenimiento (Ramírez y Maldonado, 2015). Estas limitantes refuerzan el uso de los modelos tradicionales de enseñanza a pesar del contexto tecnológico actual.

Sobre la base de las reformas y las políticas de digitalización en las instituciones educativas, las investigaciones analizan la relación entre la docencia y las TIC (Morales, Ramírez-Martinell y Excelente, 2015). En este sentido, se ha configurado un marco conceptual sin precedentes para otras épocas, pero característico de la sociedad del conocimiento actual. La revisión de la bibliografía correspondiente sugiere que las autoridades institucionales no cuestionan la relevancia de las TIC en la educación, por lo tanto, diseñan planes encaminados a garantizar el acceso, uso y apropiación de este tipo de tecnología para la formación, administración e investigación. En contextos burocráticos, la responsabilidad de operar la política de digitalización recae principalmente en los cuerpos docentes.

La investigación educativa no ha sido indiferente a esta situación provocada por la revolución tecnológica de la computadora, internet, web y software (Castells, 1999); no cuando el personal docente es un protagonista para gestionar el cambio de paradigma educativo. Este 
interés motivó a Afanador (2015) a realizar un estudio cualitativo con 183 docentes que se adscriben al sector educativo público de México, con el propósito de diagnosticar la prevalencia y tendencia de docentes en el uso y apropiación de TIC a partir de los escenarios de enseñanza y aprendizaje "holístico", "tecnócrata" y "reformista", y establecer la relación entre los escenarios mencionados y la concepción de modelo pedagógico docente. Concluyó que entre el personal docente existen concepciones eclécticas de los modelos pedagógicos que integran saberes disciplinares (teóricos e instrumentales), saberes didácticos y saberes tecnológicos. Por su cuenta, Celaya, Lozano, y Ramírez (2009) realizaron un estudio cualitativo en el cual analizan cinco casos de docentes en una institución educativa mexicana de nivel medio superior, con el propósito de indagar cómo ocurre la apropiación tecnológica que incorporan recursos educativos abiertos en su práctica educativa. Se obtuvo evidencia de que valoran la utilidad de las TIC, pero no desarrollan capacidades demostrativas y aplicativas contextualizadas.

Las investigaciones sobre el tema en cuestión han llevado a la creación de modelos conceptuales para explicar de manera lógica los efectos de la irrupción inesperada de las TIC en las estructuras de la escuela y el aula (Gómez, 2011). Estos son tan solo algunos de los modelos: 1) Teoría de acción razonada, 2) Modelo motivacional, 3) Teoría del comportamiento planificado, 4) Modelo de utilización de la PC, 5) Teoría de la difusión de las innovaciones, 6) Teoría unificada de la aceptación del uso de la tecnología y 7) Modelo de aceptación tecnológica (Fernández, Vallejo y McAnally, 2015; Venkatesh et al., 2003; Venkatesh y Bala, 2008). En este estudio, el modelo de aceptación tecnológica o TAM (por sus siglas en inglés), propuesto por Davis (1989), se adopta como base teórica a fin de interpretar los resultados de forma organizada. Según Yong, Rivas y Chaparro (2010), el TAM predice el uso de las TIC, basándose en dos características principales: 1) la utilidad percibida (perceived usefulness) y 2) la facilidad de uso percibida (perceived ease of use).

El TAM se aplica a varios objetos de estudio, por esta razón fue elegido como el sustento teórico de este trabajo. Las investigaciones siguientes demuestran su capacidad de adaptación a diferentes problemáticas. Por un lado, los aludidos Yong et al. (2010) realizaron un estudio cualitativo en la Universidad Autónoma de Tamaulipas con el propósito de analizar la influencia de la cultura nacional en el público usuarios de TIC. Fueron aplicados 337 cuestionarios a docentes y personal directivo y 398 cuestionarios a estudiantes en las 23 escuelas y facultades de dicha universidad. No se confirmó la relación entre la cultura nacional y el uso de las TIC, pero se encontró que el estudiantado cambia sus percepciones sobre la utilidad y facilidad de uso de las TIC según el semestre que cursa, la formación previa y la orientación vocacional. Mientras, los grupos docentes cambian su percepción tecnológica de acuerdo con el área de los estudios de posgrado cursados y su posición en el trabajo. Por otro lado, Ramírez-Correa, Rondán-Cataluña y Arenas-Gaitrán (2010) hicieron una investigación con una muestra de 159 estudiantes de una universidad de Chile, 116 hombres y 43 mujeres, con el objetivo de explorar las diferencias de género en la adopción de la tecnología e-learning. El resultado muestra que 
doi: http://dx.doi.org/10.15359/ree.21-3.6

URL: http://www.una.ac.cr/educare

CORREO: educare@una.cr

no hay diferencia estadísticamente significativa entre hombres y mujeres. Los estudios que se han desarrollado utilizando el TAM son prolíferos, lo que lleva a abordar el planteamiento del problema con antecedentes de la viabilidad de este modelo como herramienta teórica.

El perfil docente requiere el dominio de las TIC en la sociedad del conocimiento. Esta estructura social se construye sobre la base dela innovación tecnológica yel desarrollo delaciencia, lo que pone en duda la pertinencia y la viabilidad de los métodos y las herramientas tradicionales. La escuela también se ha modernizado o intenta hacerlo a través de la introducción de las TIC en el currículo y en la administración, así que ha digitalizado sus procesos e infraestructura. El estudiantado naceo crece en las estructuras de las tecnologías de lainformacióny la comunicación, de hecho, se dice que son personas "nativas digitales", "residentes del mundo digital" o "usuarias 2.0" (Hernández, Ramírez-Martinell y Cassany, 2014), por lo tanto, tienen necesidades asociadas con el contexto temporal y espacial cuando desarrollan su trayectoria escolar. En esta coyuntura, la sociedad, la escuela y el estudiantado demandan una concepción distinta de la enseñanza, tan es así que los cuerpos docentes son conscientes de la importancia de las TIC "y que si no se forman se quedarán desfasados. Es un reto que la mayoría está abordando o piensa abordar" (Orellana, Almerich, Belloch y Díaz, 2002, p. 9).

El rol docente cambia en un ambiente favorecido por las TIC (Salinas, 2004). En los modelos educativos mediados por las TIC, el personal docente deja de ser la fuente de todo conocimiento y comienza a actuar como guía del estudiantado, facilitándole el uso de los recursos y las herramientas que necesita para explorar y construir nuevos conocimientos, es decir, se convierte en un ente gestor, consejero y mediador de los recursos de aprendizaje.

Para aplicar las TIC en el proceso de enseñanza-aprendizaje, el personal docente debe ser facilitador, asesor y gestor del conocimiento. Por lo tanto, es importante saber cómo el personal docente universitario percibe el uso de las TIC durante el proceso de enseñanzaaprendizaje y, además, saber si pone en práctica sus competencias tecnologías para el consumo, la producción o la distribución del conocimiento. De manera que las universidades necesitan conocer la "aceptación tecnológica" que sus docentes tienen con el fin de hacer un balance de los resultados de la inversión en equipamiento y construcción de instalaciones que dan soporte material a la digitalización de la formación.

A partir de las demandas de la sociedad del conocimiento, las universidades privadas de Guatemala utilizan los entornos virtuales de aprendizaje. Por lo tanto, contratan a docentes con las habilidades para utilizar las TIC. Sobre la base de un estado de conocimiento incipiente en torno a la "aceptación de las TIC" por parte de docentes de las universidades privadas de este país, se formularon los siguientes objetivos y preguntas de investigación. 


\section{Objetivos y preguntas de investigación}

El objetivo principal es analizar la aceptación de las TIC como herramientas de apoyo para la docencia en universidades privadas de Guatemala. Para ello se han formulado objetivos particulares: 1) determinar el "conocimiento", 2) la "actitud" y 3) la "practicidad" de las TIC. En otras palabras, el estudio trata de responder a tres preguntas: primera, cuál es el conocimiento; segunda, cuál es la actitud y; tercera, cuál es la practicidad de las TIC, por parte del personal docente universitario en su práctica educativa.

\section{EI TAM como sustento teórico}

El modelo de aceptación tecnológica fue desarrollado por Davis (1989) para evaluar el mercado potencial existente para guiar la producción de nuevos productos en IBM Canadá, Ltd. EI TAM surge con la finalidad de realizar medidas evaluadoras de la calidad de los sistemas de información y por la necesidad de ajustarlos a los requerimientos de las tareas a ejecutar, por lo que se constituyó como un modelo idóneo para hacer predicciones de aceptación y uso de nuevas tecnologías. El modelo utiliza escalas de medida que predicen la aceptación del público usuario a través de propiedades psicométricas que fueron adaptadas a las investigaciones que se estaban desarrollando en los años 80.

El referido TAM es el sustento de este estudio. Este modelo se basa fundamentalmente en dos conceptos básicos para la explicación del uso de las TIC: 1) la utilidad percibida de las TIC y 2) la facilidad de uso percibida. La utilidad percibida se refiere a una creencia subjetiva sobre las ventajas que esa tecnología puede tener para un individuo o colectivo (Fernández et al., 2015). Es decir, que el uso de determinada tecnología aumenta el rendimiento del público usuario o el aprovechamiento que obtiene de las tareas que realiza. Mientras, la facilidad de uso percibida de una tecnología es una creencia sobre las facilidades que puede reportar esa tecnología para las actividades de la ciudadanía en comparación con la tecnología utilizada con anterioridad. EI TAM posiciona estos dos conceptos subjetivos en el centro de su modelo explicativo (Torres, Robles y Molina, 2011). No obstante, la actitud desempeña un papel fundamental en el modelo, ya que es el vínculo de unión entre los elementos subjetivos y los elementos intencionales. En otras palabras, la utilidad percibida y la facilidad de uso percibida de las TIC determinan la actitud de los individuos respecto a esta tecnología. Y dicha actitud está en la base de la intención de uso. Esto se corrobora en la investigación de Ballinas, Arroyo y Álvarez (2014), quienes hacen un "análisis de la percepción y actitud hacia el uso de la plataforma Moodle, ... versión 2.5.3, en comparación con la versión anterior 1.9.9, por parte de los [docentes] y alumnos del Instituto Tecnológico Superior de Irapuato. Se aplicó el... TAM en ambas versiones de la plataforma Moodle.... Se comprobó que la versión 2.5.3 es útil y fácil de usar, sin embargo, existe una tendencia significativa hacia el rechazo de la misma" (p. 1). A partir de los resultados, hacen 
doi: http://dx.doi.org/10.15359/ree.21-3.6

URL: http://www.una.ac.cr/educare

CORREO: educare@una.cr

ciertas sugerencias que este Instituto debe atender como: capacitar en el uso de la plataforma al público usuario y dar a conocer la intencionalidad de uso.

\section{Diseño de la investigación}

El presente estudio cuantitativo tiene dos características: es descriptivo, porque únicamente se presentan los hechos a través de instrumentos de observación; es transversal, debido a que los datos se recolectaron en un solo momento y una sola vez (Hernández, Fernández y Baptista 2006). El estudio se desarrolló entre octubre y noviembre de 2014. Se llevó a cabo con docentes de universidades privadas de la ciudad de Guatemala. Por un acuerdo de confidencialidad se omiten los nombres de las instituciones educativas y del personal docente en cuestión. Estos grupos de docentes imparten cursos de grado y posgrado en la modalidad escolarizada; sin embargo, para las universidades de adscripción es obligatorio utilizar entornos virtuales de aprendizaje en el proceso de enseñanza-aprendizaje, razón por la cual eran idóneos para participar en estudio.

\section{Participantes}

Las universidades donde se realizó el estudio son entidades privadas, con más de 25 años experiencia y atienden esencialmente a la formáción ética, científica, profesional y técnica del estudiantado, mediante el ejercicio integrado de la docencia, la investigación y el servicio a la comunidad, fomentan la investigación como fuente de conocimiento y de progreso y trabajan en el estudio de los problemas sociales con el objeto de contribuir a su solución. Cuentan con carreras de pregrado (profesorados y licenciaturas) y postgrados (maestrías y doctorados). Además, disponen de facultades como Administración de Empresas, Ciencias de la Comunicación, Ingeniería, Humanidades, Psicología y poseen un Departamento de Innovación Educativa (Tecnología).

El proceso de muestreo utilizado para elegir a los sujetos de estudio, tanto para la validación del instrumento como para la muestra definitiva, fue a través de"sujetos participantes voluntarios" (Hernández et al., 2006, p. 562), que es una forma de muestra no probabilística que se utiliza en la investigación para elaborar conclusiones sobre casos en los que los individuos confirman su deseo de participar voluntariamente en una investigación (Hernández et al., 2006).

La población de docentes fue conformada por hombres y mujeres de 35 a 60 años, 51 con licenciatura y 37 con maestría. Fueron tomados de las bases de datos de dos universidades privadas, sumando un total de 88 docentes. A estos grupos de docentes se les explicó el propósito de la investigación, pero únicamente 38 aceptaron dar información a través del cuestionario; por consiguiente, se trabajó con una muestra no probabilística de tipo participación voluntaria (Hernández et al., 2006). 


\section{Instrumento}

Se utilizó el cuestionario de Amaya (2010) que está construido con 27 ítems, tipo Likert, agrupados en tres indicadores: "conocimiento" cuenta con 7 ítems; "actitud" 12 ítems y, "practicidad" con 8 ítems . Cada ítem estuvo conformado por 4 alternativas de respuesta: "Muy frecuentemente", “Frecuentemente", “Poco frecuente” $y$ “Nunca” (ver tabla 1).

Tabla 1: Elementos que componen la aceptación de TIC

\begin{tabular}{|c|c|c|c|c|}
\hline Dimensión & Subdimensión & Abreviatura & Ítem & Escala tipo Likert \\
\hline \multirow{27}{*}{ Aceptación de TIC } & \multirow{7}{*}{ Conocimiento } & \multirow{7}{*}{ COM } & COM 1 & \multirow{27}{*}{$\begin{array}{l}\cdot \text { Nunca } \\
\cdot \text { Rara vez } \\
\cdot \text { Frecuentemente } \\
\cdot \text { Muy Frecuentemente }\end{array}$} \\
\hline & & & $\mathrm{COM} 2$ & \\
\hline & & & COM 3 & \\
\hline & & & COM 4 & \\
\hline & & & COM 5 & \\
\hline & & & COM 6 & \\
\hline & & & COM 7 & \\
\hline & \multirow{12}{*}{ Actitud } & \multirow{12}{*}{$\mathrm{ACT}$} & ACT 1 & \\
\hline & & & ACT 2 & \\
\hline & & & ACT 3 & \\
\hline & & & ACT 4 & \\
\hline & & & ACT 5 & \\
\hline & & & АCT 6 & \\
\hline & & & ACT 7 & \\
\hline & & & ACT 8 & \\
\hline & & & АCT 9 & \\
\hline & & & ACT 10 & \\
\hline & & & ACT 11 & \\
\hline & & & ACT 12 & \\
\hline & \multirow{8}{*}{ Practicidad } & \multirow{8}{*}{ PRC } & PRC 1 & \\
\hline & & & PRC 2 & \\
\hline & & & PRC 3 & \\
\hline & & & PRC 4 & \\
\hline & & & PRC 5 & \\
\hline & & & PRC 6 & \\
\hline & & & PRC 7 & \\
\hline & & & PRC 8 & \\
\hline
\end{tabular}

Nota: Elaboración propia. 
doi: http://dx.doi.org/10.15359/ree.21-3.6

URL: http://www.una.ac.cr/educare

CORREO: educare@una.cr

Se aplicó una prueba piloto a 15 sujetos independientes del estudio, pero con características similares a la población. Se obtuvo un coeficiente de confiabilidad de 0,848 determinado por el Alfa de Cronbach; esta magnitud es "muy alta" según la escala suministrada por Ruiz-Bolivar (2002). Mientras que la validez de contenido y congruencia fue de 0.9575 que según Hernández-Nieto (2002) es "muy buena". En el procedimiento participaron 3 personas calificadoras (jueces o juezas) expertas: la primera, en el área de pedagogía; la segunda en el área de TIC y la tercera en el área de evaluación.

\section{Análisis descriptivo de datos}

Paraanalizar los datos colectados seutilizóla estadística descriptiva, un método que permite la "descripción de las variables de estudio" (Hernández et al., 2006 p. 350), específicamente a través del uso de frecuencias. La utilización de la frecuencia permitió categorizar las respuestas de los ítems en función de las subdimensiones del estudio: conocimiento, actitud y practicidad. Los resultados se presentan en tablas que contienen las preguntas y las opciones de respuesta: "muy frecuentemente", "frecuentemente", "rara vez" $y$ "nunca".

\section{Resultados}

A continuación, se presentan los hallazgos obtenidos a través de los métodos estadísticos utilizados para analizar cada una de las variables de estudio.

\section{Conocimiento}

El indicador de conocimiento está compuesto por siete ítems: COM 1 corrobora conjuntamente con sus estudiantes la veracidad de la información obtenida a través de los diferentes buscadores de internet; COM 2 maneja con exactitud las diferentes herramientas que ofrecen las TIC; COM 3 es capaz de identificar los recursos necesarios de aplicaciones informáticas para la enseñanza; COM 4, cuando selecciona material informativo tecnológico, lo revisa exhaustivamente; COM 5 está convencido de la utilidad de las TIC en la función docente; COM 6 considera que las condiciones de la institución favorecen el empleo de las TIC en la enseñanza y el aprendizaje, y COM 7 se ha interesado por aprovechar los aportes de las TIC en su acción docente. Se puede observar que la mayoría de docentes dice que tiene conocimientos de TIC. Las frecuencias de las siete variables se concentran en "frecuentemente" $y$ "muy frecuentemente". En otras palabras, este grupo de docentes dice que corroboran la información de internet a través de herramientas informáticas que se han seleccionado exhaustivamente como recursos para la enseñanza. Además, indican su convencimiento de la utilidad de las TIC porque las condiciones de la institución donde trabajan tienen la infraestructura necesaria para un rendimiento óptimo (ver tabla 2). Espinoza, Blanco y Soto (2015, p. 1) encontraron un resultado similar: "el análisis arroja que los profesores de las universidades privadas [en comparación con públicas] son los que tienen una actitud más favorable por el uso de las tecnologías de información". 
Tabla 2: Conocimiento en materia de TIC

\begin{tabular}{clccccc}
\hline Subdimensión & & Muy frecuentemente & Frecuentemente & Rara vez & Nunca & Total \\
\hline \multirow{2}{*}{ COM 1 } & Abs. & 11 & 16 & 7 & 4 & 38 \\
& $\%$ & 29 & 42 & 18 & 11 & 100 \\
\hline \multirow{2}{*}{ COM 2 } & Abs. & 9 & 17 & 10 & 2 & 38 \\
& $\%$ & 24 & 45 & 26 & 5 & 100 \\
\hline \multirow{2}{*}{ COM 3 } & Abs. & 14 & 20 & 4 & 1 & 38 \\
& $\%$ & 36 & 51 & 10 & 3 & 100 \\
\hline \multirow{2}{*}{ COM 4 } & Abs. & 14 & 17 & 5 & 2 & 38 \\
& $\%$ & 37 & 45 & 13 & 5 & 100 \\
\hline \multirow{2}{*}{ COM 5 } & Abs. & 30 & 6 & 1 & 1 & 38 \\
& $\%$ & 79 & 16 & 2 & 3 & 100 \\
\hline \multirow{2}{*}{ COM 6 } & Abs. & 24 & 10 & 3 & 1 & 38 \\
& $\%$ & 63 & 12 & 8 & 3 & 100 \\
\hline \multirow{2}{*}{ COM 7 } & Abs. & 23 & 32 & 3 & 0 & 38 \\
& $\%$ & 60 & & 8 & 0 & 100 \\
\hline
\end{tabular}

Nota: Elaboración propia.

\section{Actitud}

Elindicador de actitudestá compuesto por doceítems: ACT 1 orienta el estudiogrupalasignando tarea con el apoyo de recursos virtuales; ACT 2 guía al estudiantado hacia la búsqueda rápida de la información necesaria para dar respuesta a las tareas solicitadas; ACT3 exigeal estudiantado el empleo de buscadores de información para profundizar en los conocimientos; ACT 4 guía al estudiantado en el aprendizaje de contenidos a través de las tecnologías de información y comunicación; ACT 5 crea una situación de aprendizaje que favorece el empleo de las TIC; ACT 6 familiariza al estudiantado con el empleo de las TIC en el aprendizaje para que pueda utilizarlas cuando lo amerite; ACT 7 supervisa el uso de las TIC en el estudiantado para que las utilice correctamente; ACT 8 establece horarios en el laboratorio de computación para que todo el estudiantado pueda utilizar las TIC; ACT 9 busca ayuda de personal experto para reforzar las competencias estudiantiles en el manejo de las TIC; ACT 10 usa diferentes recursos tecnológicos para facilitar el aprendizaje de la asignatura; ACT 11 facilita en el estudiantado nuevas formas de representar la información valiéndose de las TIC, y ACT 12 guía al estudiantado hacia la elaboración de productos o materiales utilizando las TIC.

Las frecuencias de las variables tienen el mismo comportamiento en la mayoría de los casos. En otras palabras, la frecuencia más alta de las variables ACT 7, ACT 8 y ACT 9 es "rara vez", mientras que la frecuencia más alta de las nueve restantes es "muy frecuentemente" o "frecuentemente". En general, la mayoría de docentes dice que tienen la actitud para guiar a sus estudiantes en el uso adecuado 
doi: http://dx.doi.org/10.15359/ree.21-3.6

URL: http://www.una.ac.cr/educare

CORREO: educare@una.cr

de los recursos educativos basados en las TIC para realizar tareas y encontrar información. Con este fin, dicen que crean situaciones de aprendizaje mediadas por tecnologías fuera del laboratorio de computación para encontrar nuevas formas de representar la información con materiales educativos innovadores. Sin embargo, aceptan que "rara vez" supervisan el uso correcto de estas herramientas en el alumnado y "nunca" buscan el asesoramiento de personal experto en la materia (ver tabla 3). En efecto, la mayoría de docentes de universidades privadas orientan al estudiantado sobre la importancia de las TIC en su desarrollo académico (Espinoza et al., 2015).

Tabla 3: Actitud en materia de TIC

\begin{tabular}{|c|c|c|c|c|c|c|}
\hline Sub-dimensión & & Muy frecuentemente & Frecuentemente & Rara vez & Nunca & Total \\
\hline \multirow{2}{*}{ ACT 1} & Abs. & 19 & 12 & 7 & 0 & 38 \\
\hline & $\%$ & 50 & 32 & 18 & 0 & 100 \\
\hline \multirow{2}{*}{ ACT 2} & Abs. & 19 & 16 & 3 & 0 & 38 \\
\hline & $\%$ & 50 & 42 & 8 & 0 & 100 \\
\hline \multirow{2}{*}{ ACT 3} & Abs. & 15 & 17 & 3 & 3 & 38 \\
\hline & $\%$ & 39 & 45 & 8 & 8 & 100 \\
\hline \multirow{2}{*}{$\mathrm{ACT} 4$} & Abs. & 17 & 15 & 5 & 1 & 38 \\
\hline & $\%$ & 45 & 39 & 13 & 3 & 100 \\
\hline \multirow{2}{*}{ ACT 5} & Abs. & 17 & 16 & 5 & 0 & 38 \\
\hline & $\%$ & 45 & 42 & 13 & 0 & 100 \\
\hline \multirow{2}{*}{ ACT 6} & Abs. & 19 & 14 & 5 & 0 & 38 \\
\hline & $\%$ & 50 & 37 & 13 & 0 & 100 \\
\hline \multirow{2}{*}{ ACT 7} & Abs. & 11 & 11 & 14 & 2 & 38 \\
\hline & $\%$ & 29 & 29 & 37 & 5 & 100 \\
\hline \multirow{2}{*}{ ACT 8} & Abs. & 7 & 6 & 14 & 11 & 38 \\
\hline & $\%$ & 18 & 16 & 37 & 29 & 100 \\
\hline \multirow{2}{*}{ ACT 9} & Abs. & 9 & 9 & 13 & 7 & 38 \\
\hline & $\%$ & 24 & 24 & 34 & 18 & 100 \\
\hline \multirow{2}{*}{ ACT 10} & Abs. & 19 & 14 & 5 & 0 & 38 \\
\hline & $\%$ & 50 & 37 & 13 & 0 & 100 \\
\hline \multirow{2}{*}{ ACT 11} & Abs. & 16 & 18 & 3 & 1 & 38 \\
\hline & $\%$ & 42 & 47 & 8 & 3 & 100 \\
\hline \multirow{2}{*}{ ACT 12} & Abs. & 17 & 15 & 5 & 1 & 38 \\
\hline & $\%$ & 45 & 39 & 13 & 1 & 100 \\
\hline
\end{tabular}

Nota: Elaboración propia. 


\section{Practicidad}

El indicador de practicidad está compuesto por ocho ítems: PRC 1 utiliza el PowerPoint como un medio para elaborar las presentaciones con un enfoque colorido que incluye el texto; PRC 2 hace que sea atractivo el estudio de alguna temática para el estudiantado mediante presentaciones con apoyo de las TIC; PRC 3 solicita al estudiantado elaborar informe empleando el programa Word; PRC 4 utiliza el chat como herramienta de comunicación con sus estudiantes; PRC 5 invita al estudiantado a participar en videoconferencias sobre alguna temática específica; PRC 6 establece comunicación con el estudiantado a través del correo electrónico; PRC 7 publica información al estudiantado mediante un sistema de publicación sencillo vía web, y PRC 8 organiza situaciones de aprendizaje integrando información de internet. La frecuencia más alta de las variables PRC 1, PRC 2, PRC 3, PRC 6 y PRC 7 es "muy frecuentemente"; mientras la frecuencia más alta de la variables PRC 4 y PRC 5 es "rara vez" y"nunca", respectivamente (ver Tabla 4). En general, la mayoría de docentes y las docentes dicen que usan PowerPoint, Word y otras herramientas similares para desarrollar presentaciones e informes con datos de internet. Además, indican que utilizan el correo electrónico y páginas web como medios de comunicación con sus estudiantes, pero "rara vez" el chat y "nunca" las videoconferencias. De acuerdo con Guzmán (2011), los recursos didácticos utilizados por los cuerpos docentes siguen siendo la computadora, memoria USB, cañón VGA y paquetería de Office, estas tecnologías podrían ser llamadas tradicionales, debido a la frecuencia de uso en el aula. Excluyen recursos propiamente educativos tales como Wikis y WebQuests.

Tabla 4: Practicidad en materia de TIC

\begin{tabular}{rlrrrrr}
\hline Subdimensión & & Muy frecuentemente & Frecuentemente & Rara vez & Nunca & Total \\
\hline \multirow{2}{*}{ PRC 1 } & Abs. & 26 & 11 & 1 & 0 & 38 \\
& $\%$ & 68 & 28 & 4 & 0 & 100 \\
\hline \multirow{2}{*}{ PRC 2 } & Abs. & 26 & 7 & 4 & 1 & 38 \\
& $\%$ & 68 & 18 & 11 & 3 & 100 \\
\hline \multirow{2}{*}{ PRC 3 } & Abs. & 24 & 10 & 2 & 2 & 38 \\
& $\%$ & 63 & 27 & 5 & 5 & 100 \\
\hline \multirow{2}{*}{ PRC 4 } & Abs. & 9 & 7 & 15 & 7 & 38 \\
& $\%$ & 24 & 18 & 40 & 18 & 100 \\
\hline \multirow{2}{*}{ PRC 5 } & Abs. & 3 & 12 & 10 & 13 & 38 \\
& $\%$ & 8 & 32 & 26 & 34 & 100 \\
\hline \multirow{2}{*}{ PRC 6 } & Abs. & 27 & 16 & 4 & 1 & 100 \\
& $\%$ & 71 & 9 & 10 & 3 & 100 \\
\hline \multirow{2}{*}{ PRC 7 } & Abs. & 23 & 24 & 4 & 2 & 38 \\
& $\%$ & 60 & 13 & 11 & 5 & 100 \\
\hline \multirow{2}{*}{ PRC 8 } & Abs. & 19 & 34 & 13 & 1 & 38 \\
& $\%$ & 50 & & & 3 & 100 \\
\hline
\end{tabular}

Nota: Elaboración propia. 
doi: http://dx.doi.org/10.15359/ree.21-3.6

URL: http://www.una.ac.cr/educare

CORREO: educare@una.cr

\section{Discusión}

Hay que recuperar un dato como punto de partida para la discusión. Solo el 3\% de docentes considera que las condiciones de la institución no favorecen el empleo de las TIC en la enseñanza y el aprendizaje. En otras palabras, este grupo de docentes indica que cuenta con el equipamiento necesario para el desempeño óptimo de las TIC en las universidades donde trabajan. Esto denota que las universidades en cuestión son de élite. Se les llama de élite para distinguirlas de otro grupo de universidades que se dedican a la absorción de la demanda, es decir, las denominadas universidades "patito" o "de garaje", términos que se refieren a la falta de condiciones para garantizar la calidad de la educación o que no tienen un registro formal para la prestación de servicios educativos.

La actitud positiva hacia las TIC no es el problema, sino más bien la resistencia al cambio. En este y otros estudios se muestra que los grupos docentes reconocen el potencial de las TIC para la didáctica (Orellana et al., 2002; Ramírez y Maldonado, 2015); sin embargo, hay tradiciones educativas arraigadas que obstaculizan la actualización de las metodologías de enseñanza y aprendizaje en la docencia. Ramírez y Maldonado (2015) encontraron que el principal inconveniente para la integración de las TIC en el aula no es la "falta de capacitación", ni la "necesidad de infraestructura", ni tampoco el "soporte o mantenimiento", sino más bien "la resistencia de uso". Si los grupos docentes continúan resistiéndose a usar las TIC, la enorme inversión de las universidades en la construcción y mantenimiento de infraestructura e implementación de programas de capacitación no será aprovechada de manera óptima.

\section{Conclusión}

Las frecuencias han sido altas en casi todas las variables. Esto significa que el personal docente encuestado tiene aceptación por las TIC en términos de conocimientos, actitudes y practicidad de acuerdo con sus respuestas, lo que no es representativo del profesorado en el sistema universitario de Guatemala. Así lo sugiere el muestreo realizado para este estudio. Como se dijo, la población inicial fue de 88 docentes, de los cuales 38 participaron (43\%) y 50 decidieron no participar (57\%). Para explicar este hecho, se plantea la hipótesis de que los grupos docentes se sienten intimidados con la investigación educativa porque la consideran como una evaluación de sus conocimientos y, por lo tanto, quienes participan se creen con aptitudes en la materia de estudio.

Cabe destacar que los grupos docentes indican que tienen diferentes grados de conocimiento de las TIC, aunque la mayoría asegura que sabe cómo utilizar estas herramientas para la enseñanza y el aprendizaje. Por lo tanto, esta circunstancia requiere un estudio complejo para identificar no solo el conocimiento, sino también el dominio de las TIC. 
Un resultado notable en términos de actitud hacia el uso de las TIC es que estos cuerpos docentes aceptan que "nunca" buscan la asesoría de personal experto para reforzar las competencias estudiantiles en el manejo de las TIC. El asesoramiento experto es esencial para la mejora continua. Por lo tanto, es contradictorio que los grupos docentes reconozcan las potencialidades de las TIC, pero que no busquen el apoyo en el campo. Además de esta aparente contradicción, Orellana et al. (2002) añaden otra: el personal docente es consciente del cambio tecnológico, pero no cree necesario utilizar las TIC para impartir su materia.

En cuanto a la practicidad en materia de TIC, los cuerpos docentes declaran que usan con frecuencia herramientas básicas como PowerPoint, Word y correo electrónico. El dominio de la paquetería de Office de Microsoft cada vez es más frecuente en los estudios recientes sobre competencias tecnológicas de docentes (Ramírez y Maldonado, 2015). De hecho, estos indicadores han permanecido durante mucho tiempo en la medición de competencias informáticas e informacionales, debido al monopolio de software que ha mantenido Microsoft en Latinoamérica. En este sentido, es oportuno abrir una línea de investigación sobre el uso de herramientas especializadas en torno a las disciplinas que ostentan y las asignaturas que imparten, tal como se ha hecho en otros países (Morales et al., 2015).

\section{Referencias}

Afanador, H. A. (2015). Diagnóstico sobre el uso y apropiación de TIC en docentes de la Secretaría de Educación Distrital. Recuperado de http://www.virtualeduca.info/ponencias2013/102/ DiagnsticosobreelusoyapropiacindeTICendocentes.pdf

Amaya, L. (2010). Tecnologías de la información y comunicación utilizadas por los docentes universitarios (Tesis de grado). Universidad del Zulia. Venezuela, Maracaibo. Recuperado de http://tesis.luz.edu.ve/tde arquivos/148/TDE-2011-06-28T10:51:43Z-1228/Publico/ CONTENIDO.pdf

Ballinas, M. G., Arroyo, M. y Álvarez, D. A. (2014). Modelo de aceptación tecnológica: Análisis comparativo entre la Plataforma Moodle SAE versión 2.5.3 y la versión 1.9.9. Revista Iberoamericana de Producción Académica y Gestión Educativa, 1, 1-25.

Castells, M. (1999). La era de la información: Economía sociedad y cultura. La sociedad red (Vol. 1). México: Siglo XXI.

Celaya, R., Lozano, F. G., y Ramírez, M. S. (Septiembre, 2009). Apropiación tecnológica en los profesores que incorporan recursos educativos abiertos (REA) en educación media superior (pp. 1-12). En Memorias del X Congreso Nacional de Investigación Educativa, Veracruz. México. Recuperado de http://www.comie.org.mx/congreso/memoriaelectronica/v10/ pdf/area tematica 07/ponencias/0295-F.pdf 
doi: http://dx.doi.org/10.15359/ree.21-3.6

URL: http://www.una.ac.cr/educare

CORREO: educare@una.cr

Davis, F. D. (1989). Perceived usefulness, perceived ease of use, and user acceptance of information. MIS Quarterly, 13(3), 319-340. Recuperado de https://doi.org/10.2307/249008

Espinoza, F. A., Blanco, S. C., y Soto, M. (2015). Uso y actitud de los profesores ante las TIC: Análisis de universidades públicas vs privadas del Sur de Sonora. Revista Iberoamericana de Producción Académica y Gestión Educativa, 02, 1-13. Recuperado de http://www.pag. org.mx/index.php/PAG/article/viewFile/280/326

Fernández, K., Vallejo, A. y McAnally, L. (2015). Apropiación tecnológica: Una visión desde los modelos y las teorías que la explican. Perspectiva Educacional, Formación de Profesores, 54(2), 109-125. Recuperado de http://www.redalyc.org/articulo.oa?id=333339872008

Gómez, J. J. (2011). La irrupción inesperada. Las tecnologías de información y comunicación en los procesos de enseñanza de educación. Madrid: Editorial académica española.

Guzmán, F. J. (2011). El uso de las nuevas tecnologías de la información y comunicación en el proceso de enseñanza-aprendizaje. En Memorias delX CongresolnternacionaldeEducación. Currículo (pp. 1-14). Tlaxcala. México. Recuperado de http://posgradoeducacionuatx.org/ congreso/?page id $=98$

Hernández, D., Ramírez-Martinell, A., y Cassany, D. (2014). Categorizando a los usuarios de sistemas digitales. Pixel-Bit. Revista de Medios y Educación, 44, 113-126. Recuperado de https://doi.org/10.12795/pixelbit.2014.i44.08

Hernández, R., Fernández, C. y Baptista, P. (2006). Metodología de la investigación. México: McGraw-Hill.

Hernández-Nieto, H. (2002). Contribuciones al análisis estadístico: Sensibilidad (estabilidad y consistencia) de varios coeficientes de variabilidad relativa y el coeficiente de variación proporcional (Cvp). El coeficiente de validez de contenido (Cvc) y el coeficiente Kappa, en la determinación de la validez de contenido según la técnica de juicio de experos. Venezuela: Universidad de Los Andes.

Instituto Nacional de Estadística Guatemala (2015). Caracterización. República de Guatemala. Recuperado de https:/www.ine.gob.gt/sistema/uploads/2014/02/26/ L5pNHMXzxy5FFWmk9NHCrK9x7E5Qqvvy.pdf

Kats, R. (2009). El papel de las TIC en el desarrollo. Propuesta de América Latina los retos económicos actuales. Madrid: Ariel. Recuperado de http://cedoc.infd.edu.ar/upload/El Papel de las TIC en el Desarrollo Katz.pdf 
Morales, A.T., Ramírez-Martinell, A. y Excelente,C.B. (2015). Apropiación de las TIC en la educación superior: Una mirada desde la disciplina del profesorado. Research in Computing Science, 108, 45-53. Recuperado de http://www.rcs.cic.ipn.mx/rcs/2015 108/Apropiacion\%20 de\%20las\%20TIC\%20en\%20la\%20educacion\%20superior \%20una\%20mirada\%20 desde\%20la\%20disciplina\%20del\%20profesorado.pdf

Orellana, N., Almerich, G., Belloch, C. y Díaz, I. (2002). La actitud del profesorado ante las TIC: Un aspecto clave para la integración. Recuperado de https://www.academia. edu/427727/La Actitud Del Profesorado Ante Las TIC Un Aspecto Clave Para La Integraci\%C3\%B3n

Ramírez, M. y Maldonado, G. A. (2015). El uso de TIC y la percepción del profesor universitario. IJERI: International Journal of Educational Research and Innovation, 5, 195-208. Recuperado de https://www.upo.es/revistas/index.php/IJERI/article/view/1628/1328

Ramírez-Correa, P., Rondán-Cataluña, F. J., y Arenas-Gaitán, J. (2010). Influencia del género en la percepción y adopción de e-Learning: Estudio exploratorio en una universidad chilena. Journal of Technology Management y Innovation, 5(3), 129-141. doi: https://doi.org/10.4067/ $\underline{\text { S0718-27242010000300010 }}$

Ruiz-Bolívar, C. (2002). Instrumentos de investigación educativa. Procedimientos para su diseño y validación. Venezuela: Ediciones CIDEG.

Salinas, J. (2004). Innovación docente y uso de las TIC en la enseñanza universitaria. Revista Universidad y Sociedad del Conocimiento, 1(1), 1-16. Recuperado de http://www.uoc. edu/rusc/dt/esp/salinas1104.pdf

SITEAL. (2014). Informe sobre tendencias sociales y educativas en America Latina. Políticas TIC en los sistemas educativos de América Latina. Recuperado de http://www.siteal.iipe-oei.org/ sites/default/files/siteal informe 2014 politicas tic.pdf

Tobar, L. A. (2010). La educación a distancia en Guatemala. Realidades y tendencias. En P. Lupion y C. Rama (Coords.), La educación superior a distancia en América Latina y el Caribe. Realidades y tendencias (pp. 98-116). Brasil: Editora Unisul. Recuperado de http://virtualeduca. org/documentos/observatorio/oevalc 2010 (tendencias).pdf

Torres, C., Robles, J. M. y Molina, Ó. (2011). ¿Por qué usamos las tecnologías de la información y las comunicaciones? Un estudio sobre las bases sociales de la utilidad individual de internet. Revista Internacional de Sociología, 69(2), 371-392 doi: https://doi.org/10.3989/ $\underline{\text { ris.2010.01.15 }}$

Venkatesh,V.yBala,H.(2008). Technology acceptance model 3 and a research agenda on interventions. Decision Sciences, 39(2), 273-312. doi: https://doi.org/10.1111/j.1540-5915.2008.00192.x 
doi: http://dx.doi.org/10.15359/ree.21-3.6

URL: http://www.una.ac.cr/educare

CORREO: educare@una.cr

Venkatesh, V., Morris, M. G., Davis, G. B., \& Davis, F. D. (2003). User acceptance of information technology: Towards a unified view. MIS Quarterly, 27(3), 425-478. Recuperado de https:// nwresearch.wikispaces.com/file/view/Venkatesh+User+Acceptance+of+Information+Te chnology+2003.pdf

Yong, L. A., Rivas, L. A. y Chaparro, J. (2010). Modelo de aceptación tecnológica (TAM): Un estudio de la influencia de la cultura nacional y del perfil del usuario en el uso de las TIC. Revista Innovar, 20(36), 187-203. Recuperado de http://revistas.unal.edu.co/index.php/innovar/ article/view/29202/29453 